ESJ Humanities

\title{
Problématique de l'apport des Migrants Internationaux au Développement Local de Batoufam à l'Ouest-Cameroun
}

\author{
Lines Mipo Tchazi \\ Doctorante, Département de Géographie, FLSH, \\ Université de Dschang, Cameroun \\ Nadine Yemelong Temgoua \\ Chargée de Cours, Département de Géographie, ENS, \\ Université de Bamenda, Cameroun \\ Martin Kuete \\ Professeur Émérite des Universités
}

Doi:10.19044/esj.2021.v17n6p88

Submitted: 19 December 2020

Accepted: 20 January 2021

Published: 28 February 2021
Copyright 2021 Author(s)

Under Creative Commons BY-NC-ND

4.0 OPEN ACCESS

Cite As:

Tchazi M.L., Temgoua Y.N. \& Kuete M. (2021). Problématique de l'apport des Migrants Internationaux au Développement Local de Batoufam à l'Ouest-Cameroun. European Scientific Journal, ESJ, 17(6), 88. https://doi.org/10.19044/esj.2021.v17n6p88

\section{Résumé}

L'étude portant sur « la problématique de l'apport des migrants internationaux au développement local de Batoufam à l'Ouest-Cameroun», s'inscrit dans la relation migration internationale/développement qui est au cœur de nombreux débats en Sciences Sociales. Plusieurs études ont montré et démontré que nonobstant la distance physique entre leurs pays d'origine et celui d'accueil, les migrants internationaux sont des acteurs du développement local via leurs différentes interventions. Cependant, la politique actuelle d'intervention des migrants internationaux souffre de nombreuses faiblesses. Cette étude a pour objectif d'analyser ces différentes faiblesses. Elle s'appuie sur des enquêtes par questionnaires auprès de 50 migrants internationaux, 226 personnes pour la population locale, des entretiens avec les administrateurs locaux et l'observation directe. Comme résultats, plusieurs interventions des migrants ne sont toujours pas en adéquation avec les besoins prioritaires de la population locale. $47 \%$ de la population locale interrogée affirment qu'il y a inadéquation entre les projets de développement initiés par les migrants et leurs attentes ponctuelles. $41 \%$ affirment que l'adéquation est partielle. Cette population locale pense que l'inadéquation est due au fait qu'elle soit 
marginalisée dans la mise sur pied des projets de développement. Non seulement elle n'est pas consultée avant l'initiation d'un projet, mais aussi elle n'y participe presque pas. Il arrive parfois que les interventions des migrants soient une source de conflits entre les migrants et les autres acteurs locaux du développement local. L'implication des migrants en zone de départ est très souvent aussi la source de nouveaux besoins pour la population locale (dépossessions foncières). L'on note également la dépendance financière et matérielle des bénéficiaires vis-à-vis des donneurs. Ceci entraine des répercussions sur la production nationale. Toutes ces faiblesses conduisent à une faible appréciation des interventions des migrants et une faible visibilité du migrant comme acteur à part entier du développement local.

Mots-clés : Apport, développement local, migrants internationaux, OuestCameroun (Batoufam)

\title{
Issues of the Contribution of International Migrants to the Local Development of Batoufam in Western-Cameroun
}

\author{
Lines Mipo Tchazi \\ Doctorante, Département de Géographie, FLSH, \\ Université de Dschang, Cameroun \\ Nadine Yemelong Temgoua \\ Chargée de Cours, Département de Géographie, ENS, \\ Université de Bamenda, Cameroun \\ Martin Kuete \\ Professeur Émérite des Universités
}

\begin{abstract}
This paper focuses on the "issues of international migrant's contribution to the local development of Batoufam in Western-Cameroon". It goes in line with the relationship between international migration and development which is the heart of many debates in Social Sciences. Several studies showed that despite the physical distance between their country of origin and their immigration country, international migrants are actors of the local development through their various interventions. The current policy of migrant's intervention is suffering from many weaknesses, and this study aims to analyze those weaknesses. It was conducted using questionnaires survey on 50 international migrants and 226 people of the local population, interview with the local authorities, and direct observation. The results show that several migrant's interventions are still not inadequate with the priority needs of the local population. $47 \%$ of the local population affirms that there is an
\end{abstract}


inadequacy between the development projects initiated by migrants and their priority needs. $41 \%$ affirms that the adequacy is partial. This population thinks that this inadequacy is due to the fact that they are marginalized in the setting up of development projects. Not only are they not consulted before the initiation of a project, but they do not also take part in it. Sometimes, migrant's interventions create conflicts between migrants and other local development actors. Migrant's implication in starting zone is also a source of new needs for the local population (land dispossessions). The financial and material dependence of beneficiaries toward donors was also noted. This has effects on the national production. All those weaknesses led to a weak appreciation of migrant's interventions and a low visibility of the migrant as an integral actor of local development.

Keywords: Contribution, international migrants, local development, WesternCameroon (Batoufam)

\section{Introduction}

Les études démographiques ont montré que le volume total des migrants dans le monde est en constante augmentation. Selon les Nations Unies, le nombre de migrants internationaux a atteint 244 millions en 2015, soit une augmentation de $41 \%$ par rapport à l'année $2000^{1}$. Le Cameroun, pays d'Afrique Centrale, est non seulement une terre d'accueil, mais aussi l'on dénombre de nombreux camerounais hors de leur pays. D'après Tabi (2009), on estime à près de 66.000 travailleurs immigrés camerounais au Gabon et en Guinée Équatoriale dont 50.000 au Gabon. Les questions de migration et développement s'accentuent davantage et constituent par ailleurs un sujet de discorde dans la société. Cette discorde s'articule autour de deux principales tendances axées d'une part sur l'apport positif des migrations sur le développement et d'autre part sur l'apport négatif des migrations sur le développement des pays d'origine. Cependant, allant au-delà de cette opposition stricte, une vision pluraliste a récemment vu le jour. Cette vision tient compte à la fois de l'agencéité et de la structure car aucune généralisation hâtive ne doit être faite. La relation entre la migration et le développement est plus complexe et moins linéaire que ne le laissent entrevoir toutes ces positions classiques ou l'optimisme institutionnel qui domine aujourd'hui. Aucune réponse simple ne peut être donnée. Il n'y a pas de lien automatique, mécanique ou déterminé entre les deux types de phénomènes. Les apports de la migration pour le développement varient au cas par cas, dans le temps et dans l'espace. Ceci dit, cette étude va dans le même sillage que cette vision

${ }^{1}$ International Migration Report 2017, Division de la population, Département des Affaires économiques et sociales (DAES), Nations Unies. 
pluraliste. Dans les régions de l'Ouest-Cameroun (Batoufam), l'on peut remarquer plusieurs interventions des migrants internationaux en faveur du développement de leurs terroirs d'origine. L'on se pose la question de savoir : Quels sont les mécanismes qui président au choix des projets à faire exécuter en zone de départ par les migrants internationaux ? L'objectif de cette étude est d'analyser les différentes faiblesses des interventions des migrants internationaux en faveur du développement local. Ces interventions sont certes visibles dans plusieurs secteurs. Cependant, elles ne sont pas toujours la solution idoine aux problèmes réels de la population bénéficiaire. L'on remarque de nombreuses faiblesses. Ceci dit, après une présentation des besoins prioritaires de la population locale (I), l'on table sur ces différentes faiblesses (II) qui ne sont pas sans conséquences (III).

\section{L'approche de terrain et les besoins prioritaires exprimés par la population locale}

\section{I.1. L'approche de terrain}

Les différentes lectures ont permis d'améliorer les connaissances sur la relation migration internationale et développement. Elles ont aussi permis de confirmer l'originalité de cette étude et de faire le tour des différentes problématiques abordées autour de cette relation. C'est l'exemple de la migration internationale comme facteur de développement et la migration internationale comme facteur de sous-développement. Les deux grands concepts autour de cette étude sont « migration internationale » et « développement local ». Comme justification de la zone d'étude, l'on souligne que la région de l'Ouest-Cameroun occupée par les Bamilékés, est caractérisée par une ancienne culture de la migration. De nombreux travaux dont ceux de Dongmo (1981), Barbier (1983), Tabapssi (1999) l'ont largement démontré. Avec la crise économique des années 1990 au Cameroun, la migration de ce peuple s'est accentuée. Il se déplace à la recherche des meilleures conditions de vie. Malgré ce départ pour une terre étrangère, ce peuple reste attaché à sa zone d'origine. Les Sawa par exemple qui constituent le groupe ethnique le plus représenté en France selon Kamdem (2007), ne sont pas aussi attachés à leurs familles que les Bamilékés. Cet attachement s'illustre par de nombreux projets de développement qu'ils y réalisent. Batoufam n'est pas en reste. La technique d'enquête utilisée a été axée sur des questionnaires : un premier pour les migrants internationaux (permettant d'évaluer les interventions des migrants) et un deuxième pour la population locale (permettant d'évaluer les besoins prioritaires de ladite population). Les enquêtes ont été menées dans 13 quartiers de Batoufam sur un total de 15 dont compte le groupement. Le choix de ceux-ci a été fait dans un premier temps par le simple critère qu'ils abritent au moins une intervention des migrants internationaux et aléatoirement dans un deuxième temps. Un total de 50 migrants internationaux dont 15 rencontrés 
sur le terrain et 35 via internet et 226 personnes pour la population locale ont fait l'objet de cette étude. Le questionnaire a été rempli pour la plupart par les migrants dans leurs différents pays d'accueil. En effet, les questionnaires leur ont été envoyés via internet. Ils les ont remplis et renvoyés par fichier-joint. Pour les autorités locales et les responsables des services publics et privés en l'occurrence le maire de la commune de Bayangam, du chef supérieur du groupement Batoufam, le président du comité de développement de Batoufam, les responsables des établissements scolaires primaires et secondaires, les médecins chefs des centres de santé, l'on a opté pour un guide d'entretien. Compte tenu du fait que le sujet traité s'accentue sur deux acteurs (migrants et population locale), l'on a utilisé deux types de données (primaires et secondaires). Deux techniques d'échantillonnage ont été utilisées à savoir : la technique d'échantillonnage boule de neige pour les migrants. Ici, le chercheur utilise ses connaissances et à partir d'une personne étudiée, il identifie la prochaine personne à étudier. Et la technique d'échantillonnage aréolaire pour la population locale. Elle consiste à sélectionner au préalable les zones précises où envoyer les enquêteurs.

Toutes les données quantitatives et qualitatives obtenues par l'entremise des questionnaires et des guides d'entretien ainsi que des lectures et de l'observation directe, ont été dépouillées et classifiées manuellement avant toute opération informatique. Pour le dépouillement informatique, l'on a fait usage du logiciel Excel suivant les principaux axes du questionnaire. Les résultats ont été traduits sous forme de tableaux, de graphes et figures. Tout ceci a permis de faire des analyses, des commentaires ou même des comparaisons. Ceci afin de démontrer l'influence des migrants internationaux dans le processus de développement de la zone d'étude. Le traitement du texte quant à lui s'est fait à l'aide du logiciel Microsoft Office Word 2007.

\section{I.2. Besoins prioritaires exprimés par la population locale}

Selon Cottin (1805), le besoin exprime le fait de se trouver dans une situation de manque. Les besoins prioritaires quant à eux, sont ceux qui jouissent d'une priorité sur les autres à un moment « $\mathrm{T} »$. Ce sont ceux que l'on qualifie comme étant les "attentes ponctuelles" de la population locale. L'on les considère comme devant être pris en compte en premier lieu. Il est clair qu'il y aura toujours des besoins. Mais si l'on veut y apporter des solutions bénéfiques et durables, on devrait commencer par les priorités ; c'est-à-dire le besoin prioritaire au moment « $\mathrm{T} »$. Dans ce cas d'étude, les attentes ponctuelles de cette population locale (secteurs prioritaires du développement) sont énumérées dans le Tableau 1 suivant : 
Tableau 1. Secteurs prioritaires du développement identifiés par la population locale (besoins prioritaires)

\begin{tabular}{|c|c|c|}
\hline Secteurs/Besoins prioritaires & $\begin{array}{c}\text { Effectifs (nombre de } \\
\text { personnes enquêtées) }\end{array}$ & Pourcentage (\%) \\
\hline Eau potable & $\mathbf{1 1 7}$ & $\mathbf{5 2}$ \\
\hline Santé & $\mathbf{3 8}$ & $\mathbf{1 7}$ \\
\hline Réseau routier & $\mathbf{3 0}$ & $\mathbf{1 3}$ \\
\hline Électricité & $\mathbf{1 5}$ & $\mathbf{7}$ \\
\hline Agriculture & $\mathbf{1 0}$ & $\mathbf{4}$ \\
\hline Commerce (Nécessité d'un marché) & $\mathbf{0 8}$ & $\mathbf{3}$ \\
\hline Éducation & $\mathbf{0 8}$ & $\mathbf{1 0 0} \%$ \\
\hline Total & $\mathbf{2 2 6}$ & \\
\hline
\end{tabular}

Sources : Enquêtes de terrain, juin 2018

Il est à noter que cette liste n'est pas exhaustive. L'on s'est limité à ceux-ci à cause de leur récurrence dans les souhaits de la population locale interrogée.

A la lecture du Tableau 1 ci-dessus, le besoin en eau potable occupe la première place (52\%). La population locale se plaint d'une insuffisance de points d'eau dans les différents quartiers. Elle déplore également la distance parcourue pour atteindre un point d'eau. Cette situation confirme la situation générale du Cameroun en matière d'accès à l'eau potable. En effet, il faut dire que l'accès à l'eau potable dans les zones rurales est un problème majeur pour le gouvernement du Cameroun. D'après un rapport de l'Organisation Mondiale de la Santé (OMS), 319 millions de personnes en Afrique Subsaharienne n'ont pas accès à une eau potable. Soit trois ménages sur quatre vont chercher de l'eau hors de leur domicile. 695 millions de personnes n'ont pas accès à des services d'assainissement de base ${ }^{2} .81 \%$ de la population totale du Cameroun n'a pas accès à l'eau potable $(58 \%$ en zone rurale et $23 \%$ en zone urbaine) (Kankeu, 2005). Un autre aspect remarquable à souligner est l'écart entre le besoin en eau potable et la santé, besoin occupant la deuxième place (17\%). Ceci montre combien le besoin d'eau est crucial sur le territoire.

En ce qui concerne ce second besoin prioritaire (santé : 17\%), les besoins exprimés de manière générale par la population locale sont: le renforcement des pharmacies en médicaments, la nécessité des groupes électrogènes et l'aménagement des points d'eau potable dans lesdits centres de santé. Quelques personnes interrogées affirment : "avoir de l'eau quand nos proches ou nous-mêmes sont hospitalisés n'est pas chose facile.» D'autres par ailleurs affirment que les médicaments coûtent très chers. 
Par rapport au troisième besoin prioritaire de réhabilitation du réseau routier (13\%), les populations déplorent le fait que : «les routes sont presque impraticables en saison de pluies.» A partir de là, il leur est difficile de retourner des champs avec les produits y récoltés.

L'électrification (7\%) occupant la quatrième place n'est pas en marge des besoins prioritaires de la population locale. Même si beaucoup a déjà été fait de manière générale, on peut encore noter des secteurs délaissés et ce particulièrement dans les zones rurales. Un groupe interrogé sur le terrain au quartier Fieuchip affirme : "c'est un véritable problème pour nous quand il faut charger nos téléphones. Nous sommes obligés d'aller dans les quartiers où l'installation a été faite afin de charger nos téléphones.» On note aussi plusieurs établissements scolaires n'ayant pas d'électricité en leur enceinte.

Cinquièmement, vient le besoin prioritaire des subventions dans le domaine de l'agriculture (4\%) qui se trouve être la principale occupation de la population rurale. Un agriculteur pouvait affirmer : «Nous voulons des engrais pour nos travaux champêtres, mais ceux-ci cô̂tent très chers. Si l'État et nos enfants de l'étranger pouvaient nous aider dans ce domaine, nous les agriculteurs, serons très contents.»

En sixième lieu, vient le besoin prioritaire de l'aménagement d'un marché dans la localité (4\%). Les populations se plaignent de ne pas pouvoir aisément écouler les produits des champs. En effet, la localité en elle-même n'a pas de grand marché. Les populations écoulent les produits soit au niveau du péage, soit au marché de Bangoua (localité voisine).

Septièmement, l'on a l'éducation (2\%). Les besoins prioritaires exprimés sont la nécessité des bâtiments pour salles de classe, et surtout aussi du personnel enseignant. Il faut tout de même reconnaître que dans ce volet, la population applaudit l'apport considérable des migrants internationaux. Par ailleurs, elle souhaiterait substituer une partie des interventions menées dans ce secteur à d'autres tels que celui de l'eau principalement. D'où l'analyse des faiblesses des interventions des migrants internationaux en faveur du développement de leurs terroirs d'origine.

\section{Faiblesses des interventions des migrants internationaux en faveur du développement local}

\section{II.1. Des interventions des migrants pas toujours en adéquation avec les besoins prioritaires des bénéficiaires}

Les migrants internationaux investissent certes dans le développement de leurs terroirs d'origine dans divers domaines comme le présente la Figure 1 suivante. 
Figure 1. Secteurs d'interventions des migrants internationaux

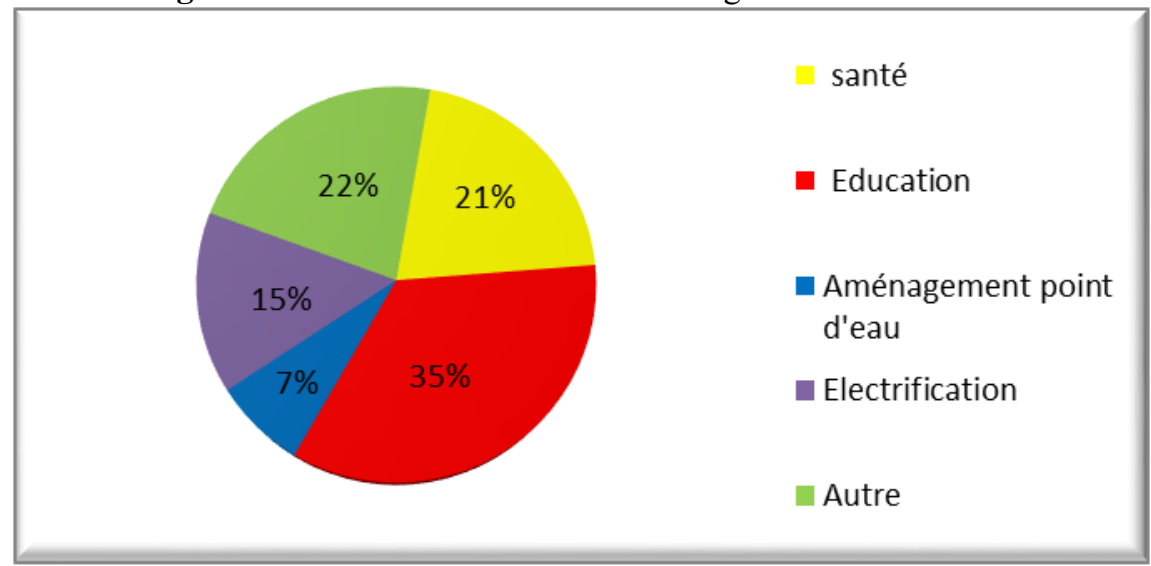

Sources : Enquête de terrain ; 2018

Il en ressort de la Figure 1 ci-dessus que les interventions des migrants internationaux sont enregistrées dans plusieurs secteurs. L'on a celui de l'éducation (35\%). On y recense l'équipement des bibliothèques, la construction des bâtiments et les dons en matériels didactiques etc. Dans le secteur santé (21\%), l'on recense la construction des centres de santé et des dons en matériels d'équipements. Au niveau de l'électrification (15\%), l'on note une extension du réseau électrique dans plusieurs maisons et les éclairages publics. L'aménagement des points d'eau (7\%) n'est pas en reste même si il reste le secteur le moins marqué par les interventions des migrants. Dans le secteur «autre », l'on recense l'aménagement de certaines routes, les interventions dans l'agriculture (dons des plants et engrais).

Seulement, plusieurs de toutes ces interventions ne sont toujours pas en adéquation avec les attentes ponctuelles des bénéficiaires comme le présente le tableau 2 suivant.

Tableau 2. Évaluation de l'adéquation entre les interventions des migrants internationaux et les besoins prioritaires de la population bénéficiaire

\begin{tabular}{|c|c|c|}
\hline Secteurs & $\begin{array}{l}\text { Interventions des } \\
\text { migrants }\end{array}$ & $\begin{array}{l}\text { Besoins prioritaires de } \\
\text { la population locale }\end{array}$ \\
\hline Eau & $7 \%$ & $51,76 \%$ \\
\hline Santé & $21 \%$ & $16,81 \%$ \\
\hline Éducation & $35 \%$ & $2,33 \%$ \\
\hline Électrification & $15 \%$ & $6,63 \%$ \\
\hline $\begin{array}{c}\text { Autres (Réseau routier, agriculture, } \\
\text { commerce) }\end{array}$ & $22 \%$ & $22,18 \%$ \\
\hline
\end{tabular}

Sources : Enquêtes de terrain, juin 2018

Le Tableau 2 ci-dessus, présente le pourcentage des interventions des migrants internationaux ainsi que celui des besoins prioritaires de la population locale. Il faut déjà noter que ce n'est pas parce que le pourcentage 
d'un secteur est supérieur au niveau des interventions et inférieur au niveau des besoins qu'il y'a forcément adéquation. Les migrants peuvent agir majoritairement dans un secteur; cependant ces interventions ne sont pas en adéquation avec les attentes ponctuelles de la population bénéficiaire.

Ceci dit, à la population locale, l'on a posé la question de savoir si les projets financés ou mis sur pied par les migrants internationaux sur leur territoire étaient en adéquation avec leurs attentes ponctuelles. La Figure 2 suivante présente les différentes réponses à l'issu de ladite question :

Figure 2. Réponses de la population locale à la question de savoir si les projets de développement mis sur pied par les migrants internationaux sont en adéquation avec leurs attentes ponctuelles.

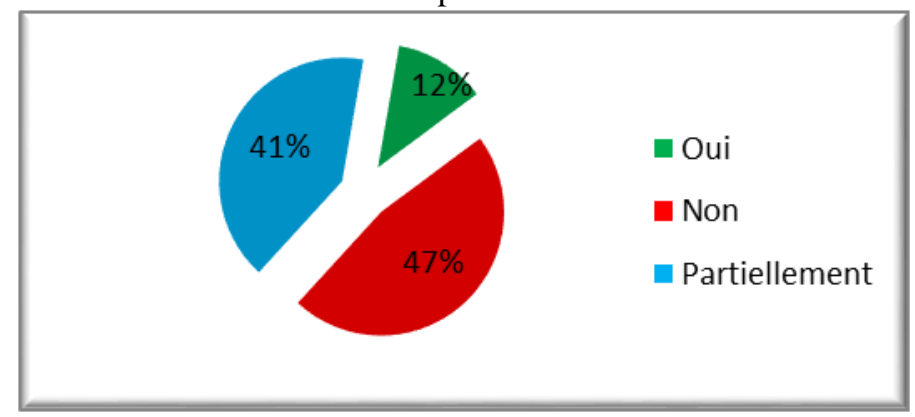

Sources : Enquête de terrain ; 2018

A la lecture de cette Figure 2 ci-dessus, l'on remarque que 12\% seulement des personnes interrogées répondent par l'affirmative à la question de savoir si les interventions des migrants sont en adéquation avec leurs attentes ponctuelles. Tandis que $47 \%$ répondent par la négation. $41 \%$ laissent comprendre que ces interventions des migrants correspondent partiellement à leurs besoins. Dans le but de comprendre cette inadéquation, à cette même population, l'on a posé la question de savoir pourquoi les interventions des migrants internationaux ne sont pas en adéquation avec leurs attentes. La Figure 3 suivante présente les différentes réponses obtenues. 
Figure 3. Les déterminants de l'inadéquation entre besoins des populations locales et interventions des migrants

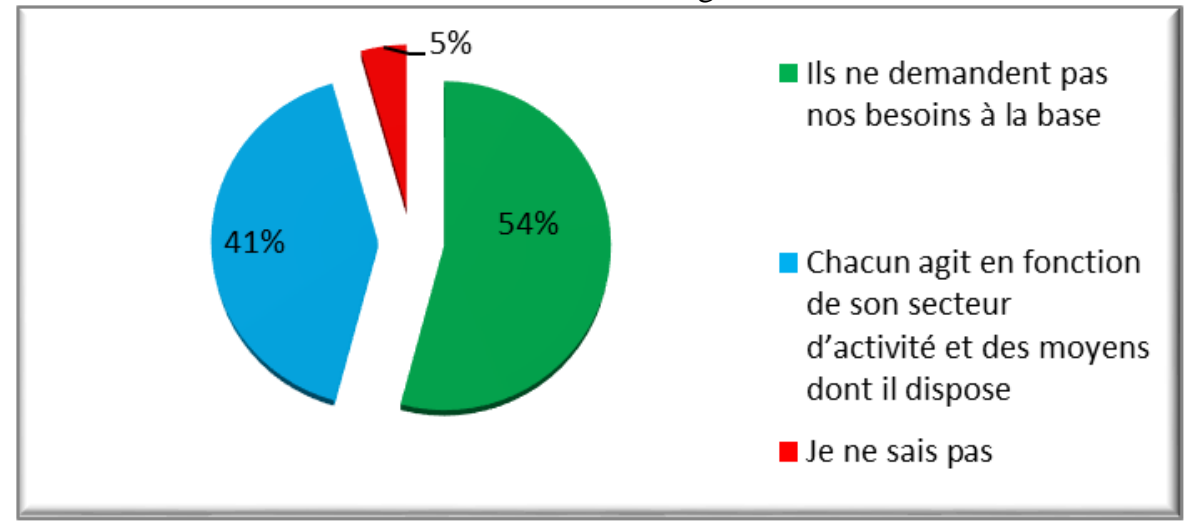

Source : Enquête de terrain ; 2018

A partir de la Figure 3 ci-dessus, pour la population bénéficiaire, les raisons de cette adéquation limitée sont la résultante d'une marginalisation (54\%). La population locale trouve qu'elle est marginalisée dans les projets de développement initiés par les migrants. En effet, leurs avis ne sont pas pris en compte avant la mise sur pied d'un projet de développement quelconque. Autrement dit, il n'y a pas un diagnostic des besoins de ladite population à la base. $41 \%$ de cette population pensent que cette situation est due au fait que les migrants agissent en fonction de leur secteur d'activités et des moyens dont ils disposent dans le pays d'accueil. 5\% affirment n'avoir aucune idée.

De manière générale, la population locale déplore l'adéquation limitée entre les interventions des migrants et leurs attentes ponctuelles. Les propos du médecin chef d'un centre de santé avec qui l'on a eu un entretien donne d'amples explications:

«Ce que nous déplorons avec nos enfants de l'étranger qui nous viennent en aide est qu'ils mènent des actions dans le village sans toutefois diagnostiquer les différents problèmes de la population. Nous reconnaissons qu'ils font beaucoup pour le village, mais il est nécessaire que ces migrants cherchent à connaître les besoins de la population avant toutes interventions dans le village afin que nous en tirions plus profit.»

Cette faiblesse d'inadéquation est visible dans plusieurs secteurs sur le territoire.

\section{Secteur eau}

Dans le Tableau 1 ci-dessus (cf. p 5), l'on constate que le secteur le moins marqué au niveau des interventions des migrants internationaux se décline en premier rang au niveau des besoins prioritaires exprimés par la population locale. Il s'agit du secteur eau (aménagement et réhabilitation des points d'eau potable) évalué à $7 \%$ au niveau des interventions des migrants et 
à $52 \%$ au niveau des besoins prioritaires de la population locale. Le décalage est considérable. En effet, pendant les enquêtes et entretiens, l'on a fait le constat selon lequel le manque d'eau potable est un problème crucial sur le territoire en générale et dans certains villages en particulier. Ce constat justifie $\mathrm{d}$ 'ailleurs les paroles du chef supérieur du village Batoufam : «Le principal problème qui touche vivement la population Batoufam est celui du besoin en eau. Nous n'avons pratiquement pas d'eau dans ce village. Même s'il y a quelques points d'eau aménagés par mes fils de l'étranger dans le village, cela reste très insuffisant.»

Cette situation de raréfaction d'eau a éveillé une curiosité conduisant à pousser les recherches dans ce secteur pour amples informations. C'est ainsi que la distance parcoure par les populations pour atteindre un point d'eau potable a fait l'objet d'une attention particulière (figure 4 suivante).

Figure 4. Distance parcourue par la population pour atteindre un point d'eau

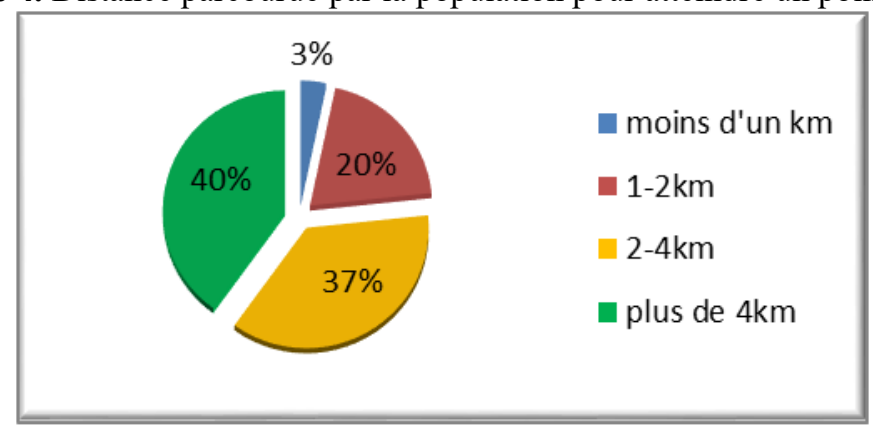

Source : Enquête de terrain ; 2018

Cette Figure 4 montre que très peu sont ceux qui parcourent moins d'un kilomètre pour atteindre un point d'eau (3\%). Plusieurs parcourent plus de 4 kilomètres (40\%). Lors des enquêtes, l'on a interrogé des personnes qui affirmaient parcourir plus de 8 kilomètres pour se procurer de l'eau à boire. Elles précisent qu'elles parcourent ces kilomètres particulièrement en saison sèche. En saison de pluies, elles boivent les eaux de pluies et parfois même celles des puits. La Figure 5 suivante donne quelques précisions sur le mode d'approvisionnement en eau potable de la population locale. 
Figure 5. Mode d'approvisionnement en eau potable de la population locale

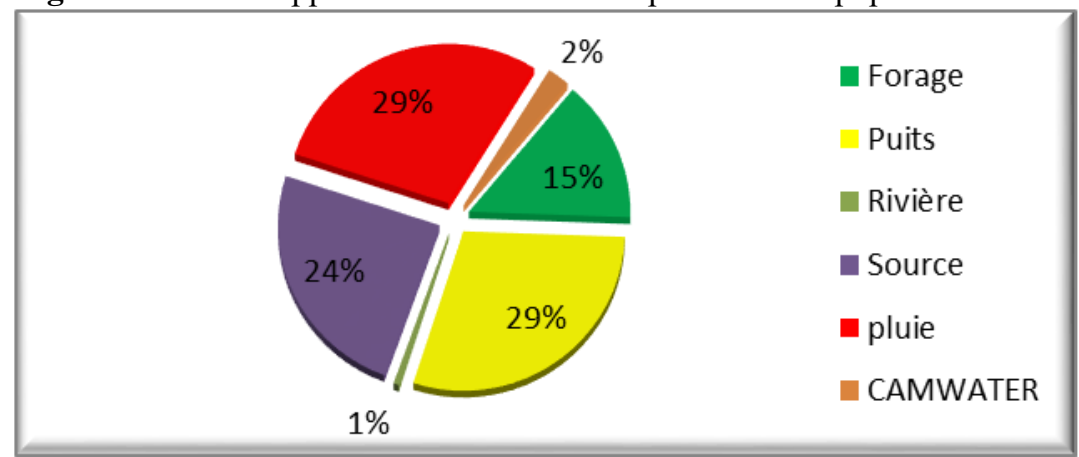

Source : Enquête de terrain ; 2018

A partir de cette Figure 5, les modes d'approvisionnement en eau potable les plus représentés sont les eaux recueillies des pluies (29\%) et des puits (29\%). suivit des eaux recueillies des sources (24\%). Les eaux recueillies au niveau des forages et celles des rivières représentent respectivement $15 \%$ et $1 \%$. Ces habitants affirment d'ailleurs que ces eaux de puits ne sont pas traitées.

Cette raréfaction d'eau potable est par conséquent à l'origine de plusieurs maladies hydriques dont souffre la population locale comme l'élucide la Figure 6 ci-dessous. Un chef de quartier pouvait affirmer : "Nous avons besoin d'eau à boire dans cette localité. Nous souffrons régulièrement ici ; moi particulièrement j'ai un problème d'amibes à cause de la mauvaise qualité d'eau que nous buvons.»

Figure 6. Maladies dont souffre très souvent la population locale

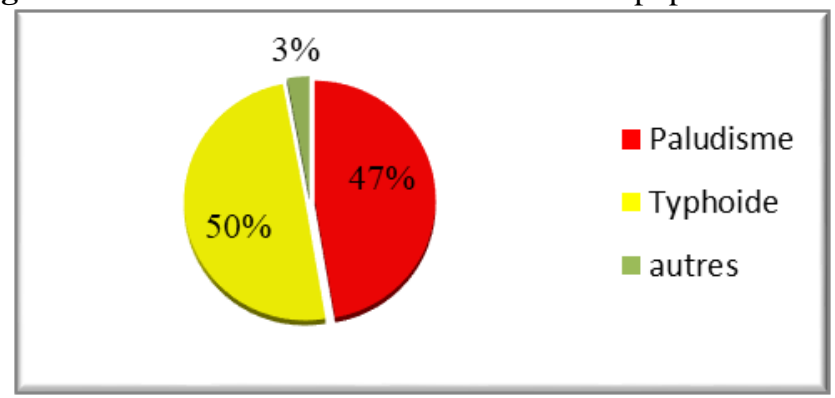

Source : Enquête de terrain ; 2018

L'on constate de la Figure 6 ci-dessus que la fièvre typhoïde occupe la première place avec un pourcentage de $50 \%$. En suite vient le paludisme $(47 \%)$. Dans le secteur «autres », l'on a le rhumatisme articulaire et la diarrhée (3\%). Ceci justifie les propos du médecin chef de l'hôpital intégré du groupement: «Les maladies récurrentes dans notre structure sont principalement la typhoïde et le paludisme. Ceci est dû en grande partie à la mauvaise qualité des eaux que boive la population même s'il y'a d'autres 
causes et ce particulièrement en ce qui concerne le paludisme.» Les infirmiers chefs de plusieurs centres de santé affirment que l'une des maladies fréquentes au sein de leurs structures est la typhoïde. Ceci à cause de la mauvaise qualité des eaux dont les populations boivent. L'un affirme : «Les habitants boivent régulièrement les eaux de pluies et de puits.» Le secteur sanitaire souffre aussi de cette faiblesse d'inadéquation.

\section{Secteur sanitaire}

Dans le domaine de la santé, les migrants internationaux font des prouesses dans leurs terroirs d'origine. Cependant, dans certains centres de santé où l'on a enregistré leurs interventions, elles ne sont toujours pas en adéquation avec les besoins réels desdits centres. L'on a par exemple constaté dans un centre de santé certains dons de machines qui n'ont jamais été utilisés (planche 1 suivante). Le responsable dudit centre affirmera d'ailleurs : «Nous avons reçu certaines machines des fils du groupement de l'étranger; ils sont là depuis un bon bout; non seulement nous ne savons pas l'utiliser, mais plus encore nous ne maîtrisons vraiment pas son utilité.»

Planche 1. Machines jamais utilisées dans le centre de santé intégré du groupement

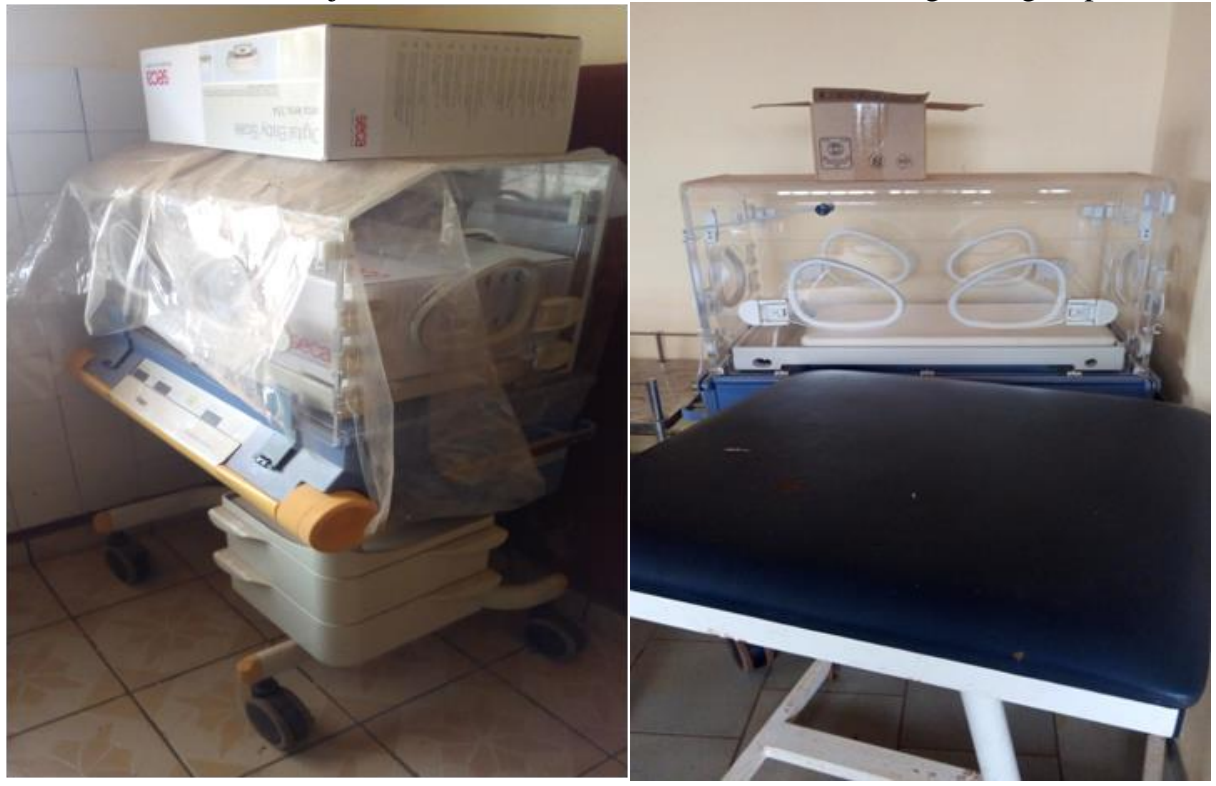

Sources : Auteur, juin 2018

L'on a également repéré des centres de santé ayant reçus des dons en médicaments. Cependant, parmi ces médicaments plusieurs sont hors d'utilisation. Le personnel de plusieurs centres de santé reconnaît les multiples interventions des migrants internationaux dans leurs différentes structures. Mais il déplore le fait que plusieurs de ces interventions ne rencontrent vraiment pas les besoins ponctuels desdits centres. La photo 1 suivante par 
exemple illustre un centre de santé qui bien évidemment a reçu certains dons en médicaments et d'équipements. Mais l'on déplore dans ledit centre de santé le manque d'eau. Il fonctionne en réalité avec un puits qui a été aménagé par les riverains.

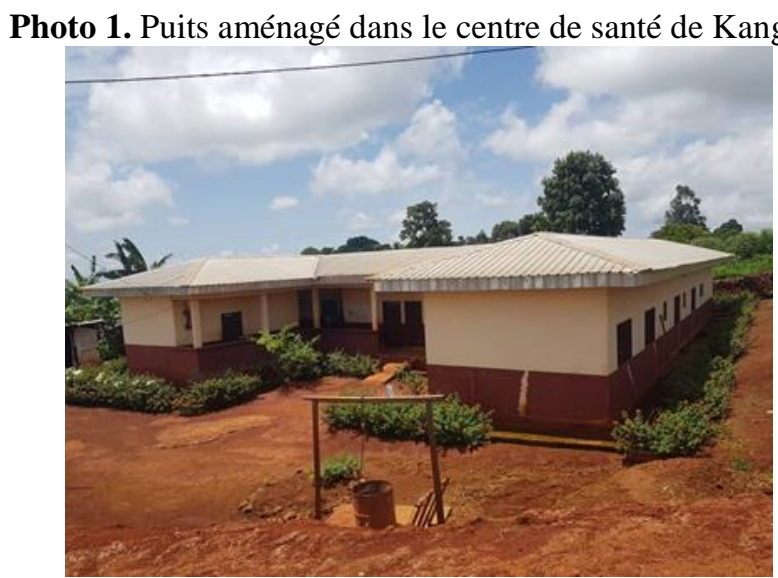

Sources : Auteur juin ; 2018

Ce problème d'inadéquation ne se limite pas au niveau de l'aménagement et/ou réhabilitation des points d'eau et la santé. L'on remarque aussi cette faiblesse au niveau de l'éducation malgré le déploiement remarquable des migrants internationaux dans ledit secteur.

\section{$>$ Secteur de l'éducation}

Dans le secteur de l'éducation de manière générale, on peut remarquer plusieurs établissements sans branchement en eau courante et en électricité. Parfois même sans toilettes fonctionnelles. Certains établissements sont sans portes et fenêtres. Dans d'autres on peut remarquer l'absence d'infirmerie et un déficit qualitatif et quantitatif du personnel enseignant.

Ceci dit, l'on a observé dans ce secteur que les interventions des migrants n'étaient pas d'une grande utilité pour les bénéficiaires. Par exemple, dans certains établissements parcourus, l'absence d'électricité est un problème crucial comme souligné ci-dessus. Cependant parmi ces mêmes établissements, on repère certains ayant reçus des dons en ordinateurs. Il est évident que ces ordinateurs ne seront d'aucune utilité à ces établissements.

D'après les enquêtes de terrain, pour une partie de la population environnante, bien qu'étant reconnaissante, elle souhaiterait substituer certaines interventions des migrants de ce secteur à un autre. Elle affirme: «Nous sommes tout de même reconnaissantes pour ce que font les migrants pour nos enfants, mais nous avons autres besoins cruciaux dans ce village comme par exemple le problème d'eau. Nous avons besoin d'eau.» 
A partir de là, l'on se rend compte que si une concertation entre toutes les parties prenantes (différents acteurs du développement local), ou alors si un diagnostic des besoins des bénéficiaires avait eu lieu avant toutes ces interventions, ces dernières auraient été réorientées de manière à être plus bénéfiques à la population bénéficiaire. Ceci conduit à une autre faiblesse qui est une relation déconnectée entre les migrants et les autres acteurs locaux de développement.

\section{II.2. Rapports conflictuels entre migrants et autres acteurs de développement local et genèse de nouveaux problèmes}

Les acteurs locaux (collectivités territoriales décentralisées, élite urbaine, comités de développement et population locale), depuis l'avènement de la crise en milieu rural camerounais, jouent un rôle principal dans le développement local. Ceci via des contributions individuelles mais surtout aussi par le biais des associations et autres structures d'encadrement (Kuete, 2001 ; Fodouop, 2003 ; Yemelong, 2007). Ceci dit, le développement local se veut être une démarche globale de mise en mouvement et en synergie des acteurs locaux pour la mise en valeur des ressources humaines et matérielles d'un territoire donné ; en relation négociée avec les centres de décision des ensembles économiques, sociaux et politiques dans lesquels ils s'intègrent (Houée, 1996).

A partir de là, l'on comprend que les migrants internationaux, dans leur élan de cœur en faveur du développement local devraient interagir avec les acteurs locaux. Mais tel n'est toujours pas le cas sur le territoire. Les rapports qu'entretiennent ces deux catégories d'acteurs du développement local (migrants internationaux et autres acteurs locaux) sont très souvent conflictuels. La Figure 7 suivante donne amples explications.

Figure 7. Nature des relations qu'entretiennent les migrants internationaux avec les autres acteurs locaux du développement

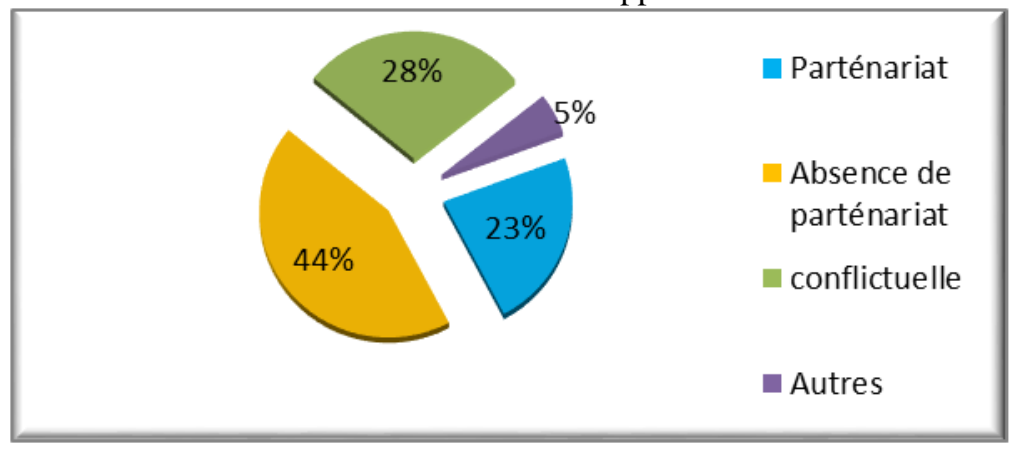

Sources : Enquêtes de terrain ; 2018

Cette Figure 7 ci-dessus présente la nature des relations qu'entretiennent les migrants internationaux avec les autres acteurs locaux. 
44\% affirment ne pas travailler en partenariat avec les acteurs locaux. 28\% affirment avoir de relations conflictuelles avec les autres acteurs locaux du développement local. Les interventions des migrants sur le territoire ne font toujours pas l'unanimité avec les autres acteurs locaux du développement. L'accent est mis particulièrement ici sur les élites urbaines qui elles aussi agissent presque dans les mêmes domaines sociaux en terme de développement. A partir d'ici, l'on peut comprendre l'analyse de Yemelong (2017), lorsqu'elle affirme que les émigrés internationaux ayant fourni d'efforts supplémentaires en traversant les limites des villes pour se rendre à l'extérieur du pays, ont besoin aussi d'une légitimé dans leurs communautés d'origine. Ceci n'est pas toujours facile dans la mesure où plusieurs membres de l'élite voient en ces émigrés internationaux des concurrents qui risqueront de leur faire ombrage. Comme exemple, l'on a le cas de la fête de l'excellence scolaire du lycée de Batoufam financée en partie par certains migrants internationaux qui a été torpillée en 2015. Selon les informations obtenues sur le terrain, cela était la résultante des conflits de leadership entre les membres du comité de développement. Cette situation influence négativement la qualité de l'impact des interventions des migrants. Outre ces conflits entre les acteurs locaux et les migrants, on peut aussi noter la naissance de certains problèmes au niveau de la population locale.

Non seulement les interventions des migrants ne sont toujours pas en adéquation avec les attentes de la population locale comme démontré cidessus, mais aussi, elles sont parfois la source de nouveaux besoins ou alors de certains problèmes pour ladite population (Figure 8).

Figure 8. Réponses de la population locale à la question de savoir si les interventions des migrants internationaux ont entrainé des problèmes nouveaux sur leurs territoires

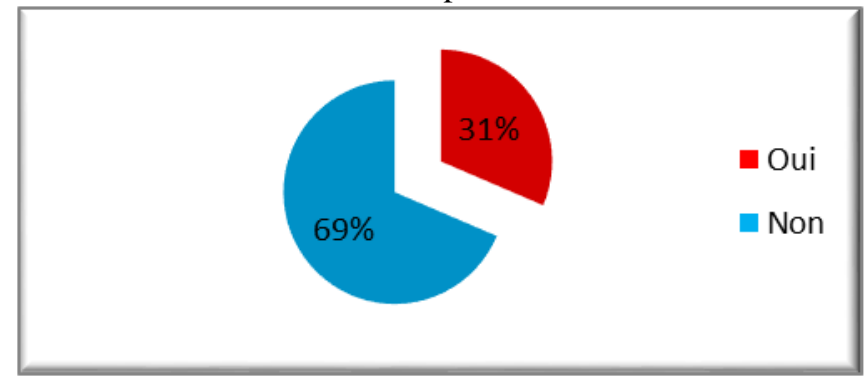

Source : Enquête de terrain ; 2018

La Figure 8 ci-dessus présente les réponses données par la population locale à la question de savoir si les interventions des migrants internationaux ont parfois entrainé des problèmes sur leur territoire. $31 \%$ répondent par l'affirmative. Comme exemple de ces problèmes, la population fait occurrence des problèmes fonciers. En effet, certains riverains se plaignent des dépossessions foncières au profit des personnes nanties parmi lesquels les migrants internationaux. Ces personnes nanties achètent les terrains des 
personnes démunies et y investissent. Ces investissements sont très récurrents dans l'immobilier et les biens de consommation. D'après la population locale, la conséquence suivante est parfois l'augmentation du coût de vie particulièrement en ce qui concerne le loyer. Plusieurs études ont montré que les migrants internationaux favorisent très souvent le processus d'urbanisation de leur pays d'origine. Ceci grâce aux différents édifices qu'ils construisent sur ces zones. Kamno (2016) affirme : «On assiste de plus en plus à la résorption des habitats précaires par des logements plus modernes. L'agrégation des constructions de maisons dans la périphérie ainsi qu'au « centre des périphéries » embellit le paysage.» Mansour (2000) de renchérir que les migrants affirment leur présence dans l'espace urbain à travers leurs constructions. Il continue en démontrant qu'en passant d'une échelle à une autre, on identifie les mutations de l'espace sous l'effet des investissements immobiliers des migrants.

Ceci dit, contrairement à ce que développent les auteurs ci-dessus mentionnés, l'on pense que s'il est clair que les constructions des migrants internationaux favorisent le processus d'urbanisation de leurs pays d'origine, elles accentuent par ailleurs aussi le phénomène de prolétarisation. Les personnes nanties achètent les terres des «pauvres» pour y investir. Le faussée entre ces deux groupes de personnes continue de s'élargir. Pendant que les personnes nanties s'enrichissent davantage, les pauvres s'appauvrissent davantage également. Or le développement local se veut être un processus qui impulse, construit et conforte les dynamiques locales et autorise une amélioration substantielle du vivre ensemble et du bien-être de tous. A partir de là, il est difficile de se limiter juste au fait que le migrant mène certaines actions dans son terroir d'origine pour le qualifier d'acteur du développement local.

Par ailleurs, un autre effet néfaste qu'entrainent ces interventions des migrants en faveur du développement de leurs terroirs d'origine est la dépendance financière et matérielle des bénéficiaires vis-à-vis des migrants ou alors de leurs interventions.

\section{II.3. Migration internationale : de la dépendance financière et matérielle à une réduction de la production au niveau local}

La dynamique migratoire dans son ensemble produit parfois des effets néfastes. Elle produit des effets d'agrégation non voulus et indésirables par les acteurs en interaction ${ }^{3}$. Il s'agit ici d'une dépendance financière et même parfois matérielle entre les migrants et les membres de leurs familles restés au pays.

${ }^{3}$ Kamno, C. (2016). « Migration et développement du pays d'origine : le cas des ressortissants des régions occidentales du Cameroun. » Thèse de Doctorat, Université de Dschang, p 275 
Sur les 226 personnes interrogées, 147 personnes ont un proche à l'étranger. A ces 147 personnes, la question de savoir si elles reçoivent de l'aide ou non de leurs proches résidant à l'étranger leurs a été posée (Figure 9).

Figure 9. Réponses de la population locale à la question de savoir si elle reçoit ou non de l'aide du proche vivant à l'étranger

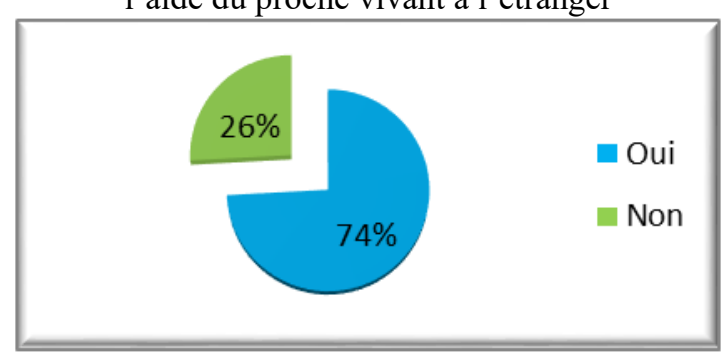

Source : Enquête de terrain ; 2018

A partir de la Figure 9 ci-dessus, sur les 147 personnes interrogées ayant un proche à l'étranger, 109 personnes $(74 \%)$ reçoivent de l'aide (financière et matérielle) de cette personne. 38 personnes (26\%) ne reçoivent pas de l'aide de leur proche parti pour l'étranger.

Ces envois des fonds des migrants internationaux à leurs différentes familles dans le pays d'origine représentent une forme d'assurance économique et un soutien remarquable. Surtout que l'on se trouve majoritairement en zone rurale. Seulement, cet apport financier entraine quelques fois chez certains bénéficiaires une dépendance économique qui de manière répétitive, influence aussi négativement la capacité de production de ces bénéficiaires. Cette situation à première analyse, semble ne pas représenter un véritable problème surtout si l'on l'observe à une échelle locale. Mais la juxtaposition de ces types de comportement peut avoir un effet péjoratif sur la production économique à l'échelle nationale ${ }^{4}$. C'est dire que les enjeux financiers de la dépendance financière peuvent se faire remarquer considérablement au niveau macroéconomique. Un migrant évoluant au canada avec qui l'on a eu un entretien pouvait affirmer : "Pour plusieurs de nos frères au pays, nous avons considérablement de l'argent. Ma famille par exemple, ne compte majoritairement que sur les fonds que je leur fait parvenir pour vivre.» Allant dans le même sillage, un chef de famille affirmera : "Je ne compte que sur ce que mon fils qui vit en Europe m'envoi à la fin de chaque mois pour vivre.»

A partir d'ici, l'on peut comprendre à quel niveau les familles peuvent êtres improductives; impactant de ce fait négativement la production au niveau national. L'on a également eu des échanges avec certains jeunes en âge de travailler. Ces derniers affirment dépendre des fonds que leur envoient leurs 
proches évoluant en pays étranger. Ceci va en droite ligne avec les travaux de Kamno (2016) effectués dans les régions occidentales du Cameroun où certains jeunes et en âge de travailler ont pris la décision d'attendre l'aide des migrants. Pour cette auteure, cette mentalité rend les jeunes improductifs. C'est également ce que les auteurs Azam et Gubert (2004) ont démontré dans leurs travaux effectués dans la région de Kayes au Mali. Pour ces auteurs, en devenant une composante permanente du budget des ménages bénéficiaires, les transferts de fonds des migrants internationaux peuvent pousser ces ménages à réduire leurs efforts de production. Autrement dit, en dehors de la satisfaction des besoins de leurs familles restées dans le pays d'origine, les économies nationales n'en tirent presque pas d'effets d'entrainement. Ce qui contribue à renforcer et à perpétuer le rapport de dépendance entre le migrant et la famille d'origine. Ceci pourrait aussi justifier cette logique de la Banque Mondiale (BM) qui pense que si les transferts des personnes vivant hors de leurs pays d'origine vers leurs pays d'origine étaient bien conduits, ils pourraient aider à réduire la pauvreté. COIFFARD (2011) de renchérir ceci dans sa thèse de Doctorat. Selon cette auteure, l'impact positif supposé des transferts de fonds des transmigrant sur la croissance et les investissements des pays bénéficiaires ne se vérifie pas. Elle pense que ces transferts ne sont pas une panacée pour le développement des pays de départ des migrants. Ils engendrent une forte dépendance des bénéficiaires qui deviennent parfois peu productifs au niveau local.

Plus loin, cette analyse va en droite ligne avec la théorie de la dépendance en étude des questions migratoires. Pour cette école de pensée, la migration internationale participe à l'élargissement de l'écart entre le centre et la périphérie. Cette situation a pour conséquence de mener à un développement passif, non productif, dépendant des transferts. Elle peut d'ailleurs accentuer les inégalités au détriment de la solidarité et de l'intégrité socio-culturelle que les communautés possédaient auparavant (UNRISD, 2007). Autrement dit, la migration internationale accentue le phénomène de pauvreté dans les pays en développement. Elle favorise les problèmes des pays qualifiés de sous-développés. Gunder (1996) qualifie ceci de: «The development of underdevelopment in Chile. » Tel est le cas à Batoufam où certaines personnes interrogées ne s'intéressent pas du tout aux interventions des migrants internationaux dans leur région. Elles affirment : «Nous sommes pauvres et n'avons personne à l'étranger qui puisse aussi penser à nous ou alors nous envoyer de l'argent et des biens.» Partant de là, on peut comprendre l'analyse de Wanner (2008). Pour cet auteur, les transferts de fonds des migrants ne contribuent pas toujours d'une manière optimale au développement. Ces transferts de fonds peuvent provoquer dans le pays des bénéficiaires des problèmes d'inégalité entre les populations. 
Analysant l'impact des transferts au Sénégal, Daffé (2008) fait une démonstration. Le soutien apporté au budget familial via les envois d'argent des migrants constitue une forme d'assurance contre la précarité des conditions de vie des bénéficiaires et l'instabilité de l'environnement macroéconomique. Mais étant donné l'importance et la régularité des transferts de fonds, comparés aux propres capacités de création de richesse et au revenu personnel d'un grand nombre de ménages bénéficiaires, ce rôle finit par installer ces derniers dans une situation de dépendance vis-à-vis de cette source de revenu. Cette situation n'encourage pas l'activité professionnelle chez les bénéficiaires. Ces derniers ont moins d'intérêt à travailler pour de faibles salaires quand l'argent reçu du proche à l'étranger leur permet de vivre $^{5}$. Cette dépendance n'est pas seulement financière, mais elle est aussi matérielle.

En effet, en ce qui concerne la nature de l'aide que reçoivent les populations locales, $46 \%$ correspondent à l'aide matérielle. Ici l'on a entre autres les vêtements, voitures, ordinateurs, téléphones, chaussures, meubles etc. Certaines personnes interrogées affirment ne plus acheter certains produits locaux ou alors les achètent rarement. Elles affirment que leurs proches à l'étranger le leurs envoient fréquemment dès que l'occasion se pointe. Ces propos d'un chef de ménage nous en disent plus : "Nous achetons rarement certains produits ici car presque tout vient de l'étranger. Même les vêtements des enfants, leur maman n'en achète que quelques uns ici, si non la plupart vient de l'étranger.»

L'accumulation de telle situation peut empiéter ou alors aura un effet néfaste sur la production nationale. Les produits nationaux sont délaissés au profit de ceux externes. Comme le démontre Kamno (2016) dans son analyse sur les effets pervers de la migration dans les régions occidentales du Cameroun, l'importation des biens peut aussi avoir un effet néfaste sur la production nationale. Elle affirme que l'agrégation des importations des vêtements de seconde main ou neufs et autres biens produit un effet de stagnation sur la consommation des biens nationaux, notamment les pagnes camerounais etc.

Toutes ces faiblesses en rapport avec l'intervention des migrants internationaux dans le développement local ne sont pas sans conséquences. L'on note la faible visibilité des interventions des migrants par la population locale et la faible visibilité du migrant en tant qu'acteur à part entier du développement local comme le démontrent plusieurs auteurs.

\footnotetext{
${ }^{5}$ Wanner, P. (2008). «L'apport des migrants au développement. Une perspective économique », dans migration et développement : Un mariage arrangé, vol $27, \mathrm{n}^{\circ} 2$.
} 


\section{Conséquences de l'inadéquation entre interventions des migrants et besoins prioritaires des bénéficiaires}

La faible adéquation entre les interventions des migrants et les besoins prioritaires de la population bénéficiaire ne sont pas sans conséquences. Elle entraine la faible visibilité ou la faible appréciation des interventions des migrants et même du migrant lui-même en tant qu'acteur du développement local.

\section{III.1. Faible appréciation des interventions des migrants par la population locale}

Découlant du fait que les besoins prioritaires de la population bénéficiaire demeurent malgré le déploiement des migrants internationaux dans différents secteurs du développement local, l'on ne perçoit plus comme il se devrait les interventions de ces derniers. Autrement dit, cette situation de mauvaise canalisation des interventions des migrants empiète la visibilité et la reconnaissance de leur élan de cœur. Elle détourne la population bénéficiaire d'apprécier et de reconnaître lesdites interventions. C'est pourquoi certains pensent que ce que font les migrants internationaux sur leurs territoires est insuffisant et doit être grandement amélioré (Figure 10).

Figure 10. Évaluation des interventions des migrants internationaux par la population locale

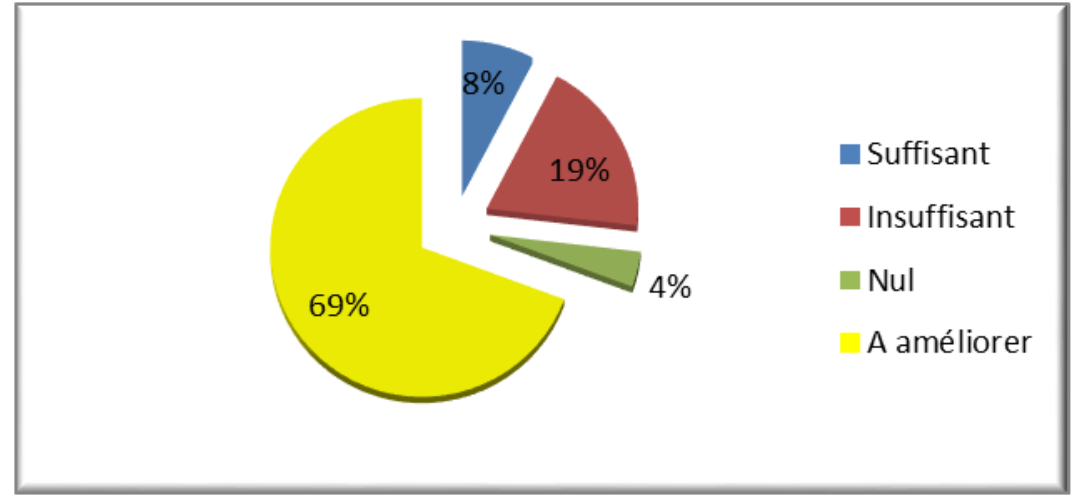

Source : Enquête de terrain ; 2018

A la lecture de cette Figure 10 ci-dessus qui présente une évaluation des interventions des migrants dans le cadre du développement local par la population locale, $19 \%$ pensent que les interventions des migrants en faveur du développement sont insuffisantes. Par conséquent, elles doivent êtres améliorées (69\%). 4\% évaluent cet apport d'être même nul. Par ailleurs, certaines (8\%) reconnaissent tout de même les efforts fournis par ces migrants dans divers domaines du développement sur leurs territoires.

L'on s'est également rendu compte d'une catégorie de personnes ne s'intéressant pas du tout aux interventions des migrants dans leur localité. Ce sont principalement des personnes n'ayant généralement aucun proche à 
l'étranger ainsi que des personnes qui ne recensent aucun projet de développement réalisé par les migrants internationaux sur leur territoire. C'est dire que les interventions des migrants sont aussi inégalement réparties sur le territoire. Pendant que certains quartiers regorgent plusieurs interventions des migrants, d'autres n'en possèdent aucune.

Cette faible visibilité ne se limite pas aux interventions des migrants, mais elle se prolonge au niveau du migrant lui-même qui fragilise son statut d'acteur du développement local.

\section{III.2. Faible visibilité du migrant en tant qu'acteur du développement local}

Comme autre conséquence de l'adéquation limitée entre les interventions des migrants et les besoins prioritaires de la population bénéficiaire, l'on remarque la faible visibilité du migrant comme étant un acteur à part entier du développement local. Si l'intervention du migrant international est faiblement perçue par les bénéficiaires, il en suit que le migrant lui-même sera faiblement perçu comme étant un acteur du développement de son terroir d'origine. L'on remarque aussi que l'intervention du migrant au lieu d'être une source d'épanouissement et d'amélioration des conditions de vie des bénéficiaires, entraine plutôt ces derniers dans une situation de conflits et de nouveaux besoins. Dans un tel contexte, le migrant ne peut être reconnu comme acteur à part entier du développement local.

L'on voudrait ici faire comprendre qu'il ne suffit pas de se limiter à la simple et unique raison que le migrant international mène des actions de développement dans son terroir d'origine pour qualifier ce dernier d'acteur du développement local. Mais bien plus il est nécessaire de se rassurer : est-ceque ce que font les migrants vont en droite ligne avec les plans de développement de leurs terroirs d'origine? Est-ce-que leurs différentes interventions sont bénéfiques à la population locale, principal bénéficiaire de leurs actions ? Ou alors sont-elles plutôt la source d'autres problèmes ? A partir de là, les migrants ne peuvent être considérés comme acteurs du développement local que si et seulement leurs différentes interventions répondent favorablement à ces quelques questions non-exhaustives ci-dessus mentionnées.

\section{Conclusion}

L'objectif de ce travail était d'analyser les faiblesses des interventions des migrants internationaux en faveur du développement local. Il en ressort des recherches que les migrants internationaux investissent certes dans le développement local de leurs terroirs d'origine dans divers domaines. Seulement, cet apport souffre de nombreuses faiblesses. Leurs interventions 
ne sont toujours pas en adéquation avec les attentes ponctuelles des bénéficiaires. La population locale déplore le fait que les interventions des migrants ne sont pas en adéquation avec leurs attentes ponctuelles (47\%). Ces interventions sont aussi parfois une source de conflits et des problèmes au niveau de la population bénéficiaire. L'on remarque des rapports conflictuels entre les migrants internationaux et les autres acteurs du développement local (collectivités territoriales décentralisées, élite urbaine, comités de développement et population locale). Une autre faiblesse est celle de la dépendance financière et matérielle des familles vis-à-vis des migrants internationaux. Ceci entraine des conséquences au niveau de la production nationale car les produits locaux sont délaissés au profit de ceux de l'étranger. De ce fait, l'on pense qu'il ne suffit pas de se limiter à la simple et unique raison que le migrant international mène des actions de développement dans son terroir d'origine pour le qualifier d'acteur du développement local. Mais bien plus, il est nécessaire de se rassurer si leurs interventions vont en étroite collaboration avec les plans de développement local de leurs terroirs d'origine. Il est aussi primordial que les migrants se rassurent que leurs interventions soient réellement bénéfiques à la population bénéficiaire et non une source de conflits et de tension pour cette dernière. Ceci nécessite une collaboration entre tous les acteurs en jeux du développement local. A partir d'ici, l'on voudrait comprendre l'origine de toutes ces faiblesses et comment y remédier.

\section{References:}

1. AFD (2013). Cadre d'intervention transversal (CIT) Migrations internes et internationales, 2010-2013, 54 p.

2. Coiffard, M. (2011). Les déterminants et impacts macroéconomiques des transferts de fonds des migrants : une analyse du cas de forte dépendance. Thèse pour l'obtention de grade de docteur de l'université de Grenoble, spécialité Sciences Économiques, université de Grenoble.

3. Daffé, G. (2008). «Les transferts d'argent des migrants sénégalais : entre espoir et risques de dépendance », in : DIOP, Momar-Coumba, Le Sénégal des migrations, mobilités, identités et sociétés, Pris : Ed. Karthala ; Dakar : CREPOS ; Nairobi : ONU-Habitat, pp105-131.

4. Deffontaines, J.P. \& Prod'homme, J.P. (2001). Territoires et acteurs du développement local, de nouveaux lieux de démocratie. Éditions de l'Aube, $179 \mathrm{p}$.

5. Dongmo, J-L. (1981). "Le dynamisme bamiléké : la maitrise de l'espace rural ».vol 1.Yaoundé, CEPER, 424 p.

6. Dongmo, J-L. (1981). Le Dynamisme Bamiléké : La Maîtrise de l'espace urbain. Vol 2. Yaoundé, CEPER, 293 p. 
7. Dumont, J-C., (2007). « Migrations et transferts de fonds. Impact sur les pays d'origine. Commentaires », Revue d'économie $d u$ développement, vol. 21, $\mathrm{n}^{\mathrm{o}}$ 2-3, pp. 189-194.

8. Evina C., et al. (2009). Migration au Cameroun : Profil national, OIM, $128 \mathrm{p}$.

9. Faini, R. (2007). «Migrations et transferts de fonds. Impact sur les pays d'origine », Revue d'économie du développement, vol. $21, \mathrm{n}^{\circ} 2$ 3, pp. 153-182.

10. Fodouop, K.F. (2003). « Citadins et développement des campagnes au Cameroun », Yaoundé, PUY, pp 39-66.

11. Gubert, F. (2009). La migration facteur de développement de la région de Kayes. Les développelement est-il une réponse aux migrations internationales ? Dossier accueillir $\mathrm{N}^{\circ} 252$, décembre, 4 p.

12. Inoussa, D. \& Zoumana, M. (2018). Impact Des Facteurs Intersubjectifs Sur L'engagement Des Cadres Ivoiriens Au Développement De Leur Localité d'Origine. European Scientific Journal, ESJ, 14(32), 237.

https://doi.org/10.19044/esj.2018.v14n32p237

13. Kamdem, P. (2009). Mutations dans les migrations camerounaises pour études en Ile-de-France : 1982-2002 in Culture et gouvernance locale, pp 80-98.

14. Kamdem, P. \& Kotlok, N. (2015). A la quête des synergies entre migrations internationales et développement local. Les éditions IRESMA, $190 \mathrm{p}$.

15. Kamno, CG. (2016). «Migration et développement du pays d'origine : le cas des ressortissants des régions occidentales du Cameroun. » Thèse de Doctorat, Université de Dschang, $480 \mathrm{p}$

16. Kankeu, R. (2005). La problématique de l'approvisionnement en eau potable dans l'agglomération de Bafoussam. Mémoire de Maitrise en géographie, Université de Dschang, $125 \mathrm{p}$.

17. Kotlok, N. \& Mimche, H. (2015). Les acteurs de la solidarité internationale : antagonisme ou complémentarité. In A la quête des synergies entre migrations internationales et développement local (KAMDEM, P. et KOTLOK, N.). Editions IRESMA, pp 97-123.

18. Kuété, M. (2001). "La ville paie sa dette envers la campagne des Hautes Terres de l'Ouest Cameroun : Transformation des paysages in les montagnes tropicales: Identités, mutation, développement». Talence, DYMSET, CRET, pp 369-381 p.

19. Mazzella, S. (2014). La sociologie des migrations. Que sais-je? Paris, PUF, n'3994, $127 \mathrm{p}$.

20. Mimché, H. \& Tourere, Z. (2009). Circulations migratoires des élites économiques dans l'Ouest du Cameroun : le cas des antiquaires. In 
Migrants des Suds (V.Baby-Collin, H. Guétat, L. Faret, G. Cortes). IRD, Éditions de l'Université de Montpellier, Presses universitaires du Mirail, 77-99 p.

21. Nassourou, I. \& Sani, M. M. (2019). La Guerre De 2011 En Libye Et Les Migrants De Retour Dans La Région De Tahoua Au Niger : Entre Incertitude Sécuritaire Et Défis De Développement Local. European Scientific Journal, ESJ, 15(35), 32.

https://doi.org/10.19044/esj.2020.v15n35p32

22. Piche, V. (2013). Les théories de la migration, Paris, éditions INED, $535 \mathrm{p}$.

23. Tabi Akono, F. (2009). Migration professionnelles. Cas du Cameroun. Communication présentée au séminaire organisé dans le cadre du partenariat pour la gestion des migrations professionnelles, Cotonou.

24. Wanner, P. (2008). «L'apport des migrants au développement : une perspective économique », Annuaire suisse de politique de développement [En ligne], Vol. 27, $\mathrm{n}^{\circ} 2 \mid$, mis en ligne le 22 mars 2010, Consulté le 26 janvier 2017. URL : http://aspd.revues.org/193.

25. Yao, K.A., Yoro, B.M., Adou Yao, C.Y., \& Amani, Y.C. (2019). Enjeux des activités humaines dans le maintien de la diversité végétale des forêts marécageuses de la Sous-préfecture de Grand-Lahou sur le littoral ivoirien. European Scientific Journal, Vol.15, No.15, pp 206228. http://dx.doi.org/10.19044/esj.2019.v15n15p206

26. Yemelong, N. (2007). Problématique de la contribution des "Élites extérieures» au développement local en milieu rural Bamiléké. Analyse du cas de Babadjou (Ouest-Cameroun) ». Thèse de Master de géographie, Université de Dschang, $153 \mathrm{p}$.

27. Yemelong, N. (2017). «Problématique de la participation des émigres internationaux camerounais au développement de leurs terroirs d'origine. Lecture à partir des zones rurales du département des Bamboutos (Région de l'Ouest) ». Thèse de Doctorat, Université de Dschang, $427 \mathrm{p}$.

28. Yves-Cyrille, N. T., \& Jérôme, A.-N. (2018). Analyse De La Contribution Des Collectivités Territoriales Au Développement Local En Côte D'ivoire : Cas Du Département D'agboville. European Scientific Journal, ESJ, 14(32), 286.

https://doi.org/10.19044/esj.2018.v14n32p286 